\title{
A real or apparent decrease in glomerular filtration rate in patients using olaparib?
}

\author{
M. A. C. Bruin ${ }^{1}$ (D) - C. M. Korse ${ }^{2}$ B. van Wijnen ${ }^{2}$ - V. M. T. de Jong ${ }^{3}$ - S. C. Linn ${ }^{3,4} \cdot$ B. van Triest ${ }^{5} \cdot$ H. Rosing ${ }^{1}$. \\ J. H. Beijnen ${ }^{1}$ - D. van den Broek ${ }^{2}$ - A. D. R. Huitema ${ }^{1,6,7}$
}

Received: 11 May 2020 / Accepted: 8 December 2020 / Published online: 14 December 2020

(C) The Author(s) 2020

\begin{abstract}
Purpose Olaparib is a poly (ADP-ribose) polymerase (PARP) inhibitor indicated for ovarian and metastatic breast cancer. Increased serum creatinine levels have been observed in patients taking olaparib, but the underlying mechanism is unknown. This study aimed to investigate if patients receiving olaparib have increased creatinine levels during olaparib treatment and whether this actually relates to a declined glomerular filtration rate (GFR).

Methods We retrospectively identified patients using olaparib at the Netherlands Cancer Institute - Antoni van Leeuwenhoek (NKI-AVL) from 2012 until 2020. Patients with at least one plasma or serum sample available at baseline/off treatment and during olaparib treatment were included. Cystatin $\mathrm{C}$ levels were measured, creatinine levels were available and renal function was determined by calculating the estimated glomerular filtration rate (eGFR) using the Creatinine Equation (CKD-EPI 2009) and the Cystatin C Equation (CKD-EPI 2012).

Results In total, 66 patients were included. Olaparib treatment was associated with a $14 \%$ increase in median creatinine from 72 (inter quartile range (IQR): 22$) \mu \mathrm{mol} / \mathrm{L}$ before/off treatment to 82 (IQR: 20$) \mu \mathrm{mol} / \mathrm{L}$ during treatment $(p<0.001)$ and a $13 \%$ decrease in median creatinine-derived eGFR from 86 (IQR: 26) $\mathrm{mL} / \mathrm{min} / 1.73 \mathrm{~m}^{2}$ before/off treatment to 75 (IQR: 29) $\mathrm{mL} / \mathrm{min} /$ $1.73 \mathrm{~m}^{2}$ during treatment $(p<0.001)$. Olaparib treatment had no significant effect on median cystatin $\mathrm{C}$ levels $(p=0.520)$ and the median cystatin $\mathrm{C}$-derived eGFR $(p=0.918)$.

Conclusions This study demonstrates that olaparib likely causes inhibition of renal transporters leading to a reversible and dosedependent increase in creatinine and does not affect GFR, since the median cystatin C-derived eGFR was comparable before/off treatment and during treatment of olaparib. Using the creatinine-derived eGFR can give an underestimation of GFR in patients taking olaparib. Therefore, an alternative renal marker such as cystatin C should be used to accurately calculate eGFR in patients taking olaparib.
\end{abstract}

Keywords Olaparib $\cdot$ Creatinine $\cdot$ Cystatin C $\cdot$ eGFR $\cdot$ Renal function

M. A. C. Bruin

ma.bruin@nki.nl

1 Department of Pharmacy \& Pharmacology, The Netherlands Cancer Institute - Antoni van Leeuwenhoek, Plesmanlaan 121, 1066 CX Amsterdam, The Netherlands

2 Department of Laboratory Medicine, The Netherlands Cancer Institute, Amsterdam, The Netherlands

3 Department of Molecular Pathology, The Netherlands Cancer Institute, Amsterdam, The Netherlands

4 Department of Medical Oncology, The Netherlands Cancer Institute, Amsterdam, The Netherlands

5 Department of Radiation Oncology, The Netherlands Cancer Institute, Amsterdam, The Netherlands

6 Department of Clinical Pharmacy, University Medical Center Utrecht, Utrecht University, Utrecht, The Netherlands

7 Department of Pharmacology, Princess Máxima Center for Pediatric Oncology, Utrecht, The Netherlands

\section{Introduction}

Olaparib is a potent poly (ADP-ribose) polymerase (PARP) inhibitor approved for the treatment of patients with ovarian cancer and patients with breast cancer (BRCA)-mutated human epidermal growth factor receptor-2 (HER2) negative metastatic breast cancer $[1,2]$. Olaparib is orally administrated at a recommended dose of $300 \mathrm{mg}$ (tablets) or $400 \mathrm{mg}$ (capsules) twice a day (BID). In patients taking the recommended dose, increased serum creatinine levels (up to 23\%) over baseline were observed [2].

Creatinine is an endogenous product of creatine metabolism in muscles and is used as a marker to calculate the glomerular filtration rate (GFR). The GFR is used as a functional parameter to evaluate renal function of patients in clinical practice. Creatinine is primarily filtered by the glomerulus, but active tubular secretion accounts 
for a substantial part of creatinine clearance as well $(10-40 \%)$ [3]. Active tubular secretion of organic compounds including drugs, toxins, and endogenous compounds is mediated by multiple solute carrier (SLC) transporters in the kidney. Active tubular secretion of creatinine is mainly driven by the organic cation transporter 2 (OCT2) expressed on the basolateral membrane and the multidrug and toxin extrusion protein (MATE) 1 and MATE2-K expressed on the apical membrane of the proximal tubule cells in the kidneys (Fig. 1a) [4]. Interaction with these transporters can cause a nonpathological increase in serum creatinine which has been reported for several drugs such as cimetidine [5], trimethoprim [6], and abemaciclib [7]. An increase in serum creatinine will impact the estimated glomerular filtration rate (eGFR) based on serum creatinine measurements without clinically meaningful alterations in glomerular filtration and renal function. Alternative endogenous markers such as cystatin $\mathrm{C}$ are available for assessing GFR and renal function. Cystatin $\mathrm{C}$ is freely filtrated by the glomerulus, completely reabsorbed and metabolized by the proximal tubule cells, not secreted by renal transporters like creatinine, and not affected by age, gender, changes in diet, or muscle mass $[8,9]$.

Evaluation of the interaction between olaparib and renal uptake and efflux transporters in vitro shows inhibition of OCT2, MATE1, and MATE2-K by olaparib at concentrations which can be clinically relevant [10]. Therefore, the reported increase in serum creatinine during olaparib treatment could be a result of inhibition of active tubular secretion (Fig. 1b) and not as a result of reduction in GFR. Since many drugs are excreted by the kidneys and dosed based on the eGFR, including olaparib itself [11], it is important to investigate whether olaparib causes a real decrease in GFR or only an increase in serum creatinine. Dose adjustments of drugs based on the eGFR using creatinine could result in underdosing of patients.

This study aimed to investigate if patients receiving olaparib have an increase in serum creatinine level after start of olaparib treatment and if this increase is reversible after treatment discontinuation. Furthermore, we examined if the induced increase in serum creatinine is likely caused by inhibition of active tubular secretion of creatinine or a reduction in GFR by using cystatin $\mathrm{C}$ as an alternative marker for calculating eGFR.

\section{Methods}

\section{Study design and study population}

We retrospectively identified patients using olaparib at the Netherlands Cancer Institute - Antoni van Leeuwenhoek (NKI-AVL) from 2012 until 2020. Institutional review board approval was obtained for this study. Patients with at least one plasma/serum sample available up to 40 days before start of olaparib treatment (baseline) and at least one plasma/serum sample up to 90 days after start of olaparib treatment (during treatment) were included. Patients without a baseline sample but with one sample during treatment and one during (temporary) discontinuation of olaparib treatment (off treatment) were included as well. Samples were considered "off treatment" after at least 3 days of discontinuation of olaparib therapy with an olaparib concentration measured below the lower limit of quantification (LLOQ). Patients received olaparib at several dose levels, according to clinical trial protocols or as prescribed by their treating physician. Plasma/ serum samples were collected for therapeutic drug monitoring as part of routine clinical care or for pharmacokinetic assessment and stored at the pharmacy or hospital biobank. We collected data from medical records on age, weight, body mass index (BMI), race, the daily dose of olaparib, tumor type, relevant comedication, creatinine, and c-reactive protein (CRP). Cystatin C levels were measured in the available samples. Renal function was determined by calculating the eGFR using the Creatinine Equation (CKD-EPI 2009) and the Cystatin C Equation (CKD-EPI 2012) as described by Inker et al. [12]. Calculations were performed using $\mathrm{R}$ statistical software, version 3.6.0 [13] and the package "nephro" [14].
Fig. 1 In the kidneys, creatinine is transported by the OCT2 transporter on the basolateral membrane into the proximal tubule cells and subsequently transported to the urine by MATE1- and MATE2-K transporters on the apical membrane (a). Inhibition of active transport of creatinine by inhibition of the OCT2, MATE1, and MATE2-K transporters by olaparib could cause an increase in creatinine in blood (b) a

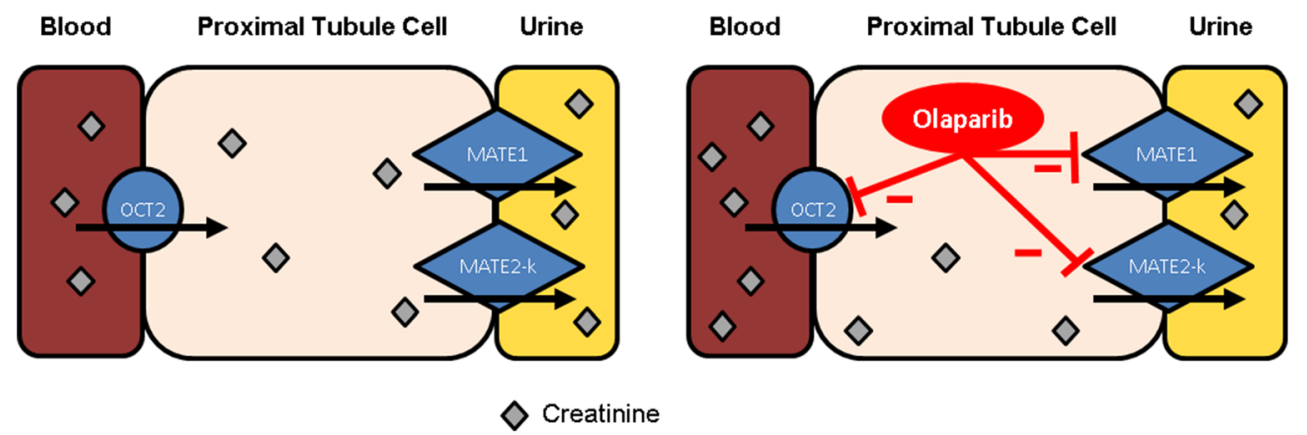




\section{Laboratory assessment}

Cystatin C was measured in serum or plasma samples at the Department of Laboratory Medicine, NKI-AVL. Analyses were performed using the Tina-quant Cystatin $\mathrm{C}$ particle enhanced immune turbidimetric assay with the Cobas 6000 analyzer (Roche, Mannheim, Germany). Latex particles coated with anti-cystatin $\mathrm{C}$ antibodies were used and aggregates with cystatin $\mathrm{C}$ were turbidimetrically determined at $546 \mathrm{~nm}$. All reagents were ready for use and the calibration range was $0.40-6.80 \mathrm{mg} / \mathrm{L}$. Creatinine was measured routinely on the day of sample collection. Creatinine assays were performed using the Cobas 6000 analyzer with an enzymatic, colorimetric method (Roche, Mannheim, Germany). The calibration range for creatinine was $5-2700 \mu \mathrm{mol} / \mathrm{L}$. A short validation was performed using plasma and serum samples from $20 \mathrm{pa}-$ tients to compare values of cystatin $\mathrm{C}$ and creatinine measured in both matrices.

\section{Statistical analysis}

Statistical analysis was performed using R statistical software, version 3.6.0 [13]. Wilcoxon signed rank tests were used to assess differences in creatinine, cystatin $\mathrm{C}$, and calculated eGFR with both equations at baseline/off treatment and during treatment of olaparib. Multiple linear regression was performed to investigate any association between covariates and change in creatinine and cystatin $\mathrm{C}$ levels.

\section{Results}

\section{Demographics}

In total, 66 patients were included of whom at least one plasma/serum sample at baseline/off treatment and one plasma/serum sample during treatment was available. Patient characteristics at baseline/off treatment are presented in Table 1 . The majority of patients $(65 \%)$ were enrolled in a clinical trial and received an olaparib dose of $25 \mathrm{mg}$ once a day (QD), $25 \mathrm{mg}$ BID or $50 \mathrm{mg}$ BID in combination with radiotherapy, with or without cisplatin. A low-dose cisplatin $\left(6 \mathrm{mg} / \mathrm{m}^{2}\right.$ on radiotherapy days (5 times a week)) was administrated to $15 \%$ of the patients. Sixteen out of 66 patients (23\%) were treated with the recommended dose of $300 \mathrm{mg}$ (tablets) or $400 \mathrm{mg}$ (capsules) BID. The 100, 200, and $250 \mathrm{mg}$ BID dose levels were a result of dose reductions due to toxicity or side effects. One patient had an impaired renal function (eGFR based on creatinine) at baseline and, therefore, received an a priori dose reduction to $200 \mathrm{mg}$ BID. CRP, potentially interesting since cystatin $\mathrm{C}$ is also an acute phase compound, was not available for most patients. Baseline eGFR values calculated with creatinine and cystatin $\mathrm{C}$ were similar.

\section{Laboratory assessment}

We collected 140 samples from 66 patients. Samples at baseline and during olaparib treatment were available from 55 patients. From 15 patients, we collected samples during olaparib treatment and after (temporary) treatment discontinuation. From 4 patients, samples at baseline, during treatment, and after treatment discontinuation were available. The same matrix was available for samples at baseline/off treatment and during treatment, except for 3 patients. During the validation, no statistical differences were found between creatinine or cystatin $\mathrm{C}$ values measured in plasma and serum; therefore, both matrices could be used to compare baseline/off treatment samples with during treatment samples. Cystatin $\mathrm{C}$ and creatinine concentrations were measured in 140 samples and all concentrations were within the calibration range.

\section{Effect of olaparib on creatinine, cystatin C, and eGFR}

Overall, olaparib treatment caused a statistically significant increase of $14 \%$ in the median creatinine level and decreased the median creatinine-derived eGFR with $13 \%$ compared to baseline/off treatment (Table 2). Stratification by dose level and separation of the baseline/off treatment group demonstrates that creatinine and the creatinine-derived eGFR levels at baseline, as well as off treatment, differ significant from levels during treatment (Table 2) in a dose-dependent manner (Table 2 and Fig. 2a and b). A non-significant increase in median creatinine level of $4 \%$ was observed at the lowest dose level of $25 \mathrm{mg}$ QD, while higher dose levels show significant increases of $8 \%$ at dose level $25 \mathrm{mg}$ BID, $12 \%$ at dose level $200 \mathrm{mg}$ BID, and $29 \%$ at dose level $300 \mathrm{mg}$ BID. Similarly, a non-significant decrease in median creatinine-derived eGFR of $4 \%$ was observed at dose level $25 \mathrm{mg}$ QD, which was significant at dose level $25 \mathrm{mg}$ BID (-12\%), $200 \mathrm{mg}$ BID $(-16 \%)$, and $300 \mathrm{mg} \mathrm{BID}(-12 \%)$. The increase in median creatinine from baseline during olaparib treatment in patients at dose level $300 \mathrm{mg}$ BID (+17\%) returned back to near baseline after treatment discontinuation $(-23 \%)$ (Table 2 and Fig. 3a). Multiple linear regression was performed to test if the dose of olaparib, sex, age, BMI, and concomitant use of cisplatin could predict the change in creatinine and creatininederived eGFR. A significant association between dose of olaparib and change in creatinine $(p=0.004)$ and creatininederived eGFR ( $p<0.001)$ was observed (Fig. 2a and b).

Overall, median cystatin $C$ levels and the median cystatin C-derived eGFR did not differ between baseline/off treatment and during olaparib treatment (Table 2). No significant differences were observed between cystatin $\mathrm{C}$ levels at baseline, during olaparib treatment, and after treatment discontinuation in patients at dose level $300 \mathrm{mg}$ BID (Table 2 and Fig. 3b). The dose of olaparib did not relate to a change in cystatin $\mathrm{C}$ 
Table 1 Baseline characteristics of all patients

\begin{tabular}{|c|c|c|}
\hline Characteristics & & Overall $(n=66)$ \\
\hline \multirow[t]{2}{*}{ Age (years) } & Median & 58.5 \\
\hline & Range & $29-80$ \\
\hline \multirow[t]{6}{*}{ Age group $(\%)$} & $21-30$ years & $2(3.0 \%)$ \\
\hline & $31-40$ years & $8(12.1 \%)$ \\
\hline & $41-50$ years & $7(10.6 \%)$ \\
\hline & $51-60$ years & $20(30.3 \%)$ \\
\hline & $61-70$ years & $17(25.8 \%)$ \\
\hline & $71-80$ years & $12(18.2 \%)$ \\
\hline \multirow[t]{2}{*}{$\operatorname{Sex}(\%)$} & Male & $23(34.8 \%)$ \\
\hline & Female & $43(65.2 \%)$ \\
\hline \multirow[t]{2}{*}{ Race $(\%)$} & White & $65(98.5 \%)$ \\
\hline & Black of African American & $1(1.5 \%)$ \\
\hline \multirow[t]{2}{*}{ Weight (kg) } & Median & 74.9 \\
\hline & Range & $51.3-132.8$ \\
\hline \multirow[t]{2}{*}{ BMI $\left(\mathrm{kg} / \mathrm{m}^{2}\right)$} & Median & 24.9 \\
\hline & Range & $19.1-44.9$ \\
\hline \multirow[t]{2}{*}{ Creatinine $(\mu \mathrm{mol} / \mathrm{L})$} & Median & 72 \\
\hline & Range & $48-185$ \\
\hline \multirow[t]{2}{*}{ Cystatin C (mg/L) } & Median & 0.91 \\
\hline & Range & $0.49-2.93$ \\
\hline \multirow[t]{2}{*}{ eGFR (creatinine) $\left(\mathrm{mL} / \mathrm{min} / 1.73 \mathrm{~m}^{2}\right)$} & Mean (SD) & $84(21)$ \\
\hline & Range & $26-123$ \\
\hline \multirow[t]{2}{*}{ eGFR (cystatin C) $\left(\mathrm{mL} / \mathrm{min} / 1.73 \mathrm{~m}^{2}\right)$} & Mean (SD) & $82(24)$ \\
\hline & Range & $18-132$ \\
\hline \multirow[t]{10}{*}{ Dose of olaparib (\%) } & $25 \mathrm{mg}$ QD tablets & $25(37.9 \%)$ \\
\hline & With cisplatin & $6(9.1 \%)$ \\
\hline & $25 \mathrm{mg}$ BID tablets & $14(21.2 \%)$ \\
\hline & With cisplatin & $4(6.1)$ \\
\hline & $50 \mathrm{mg}$ BID tablets & $46.1 \%)$ \\
\hline & $100 \mathrm{mg}$ BID tablets & $1(1.5 \%)$ \\
\hline & $200 \mathrm{mg}$ BID tablets & $5(7.6 \%)$ \\
\hline & $250 \mathrm{mg}$ BID tablets & $1(1.5 \%)$ \\
\hline & $300 \mathrm{mg}$ BID tablets & $15(22.7 \%)$ \\
\hline & 400 mg BID capsules & $1(1.5 \%)$ \\
\hline \multirow[t]{5}{*}{ Tumor type (\%) } & Mamma carcinoma & $18(27.3 \%)$ \\
\hline & Ovarium carcinoma & $12(18.2 \%)$ \\
\hline & Larynx carcinoma & $7(10.6 \%)$ \\
\hline & Oropharynx carcinoma & $1(1.5 \%)$ \\
\hline & NSCLC & $28(42.4 \%)$ \\
\hline
\end{tabular}

$B M I$, body mass index; $e G F R$, estimated glomerular filtration rate; $n$, number of subjects; $S D$, standard deviation; $Q D$, once a day; $B I D$, twice a day; NSCLC, non-small cell lung carcinoma $(p=0.917)$ and cystatin C-derived eGFR $(p=0.882)$ (Fig. $2 \mathrm{c}$ and $\mathrm{d})$.

Two patients showed a substantial change in renal function as measured with cystatin $\mathrm{C}$ and cystatin $\mathrm{C}-$ derived eGFR. Along with these changes, creatinine and creatinine-derived eGFR were changed as well. One patient at dose level $100 \mathrm{mg}$ BID (change in creatinine, creatinine-derived eGFR, cystatin $\mathrm{C}$, and cystatin $\mathrm{C}-$ derived eGFR of $+19 \%,-18 \%,+22 \%$, and $-19 \%$, respectively) had progression of abdominal metastases and problems urinating at moment of off treatment sample collection. One patient at the $400 \mathrm{mg}$ BID dose level (change in creatinine, creatinine-derived eGFR, cystatin $\mathrm{C}$, and cystatin C-derived eGFR of $-14 \%,+20 \%$, - 


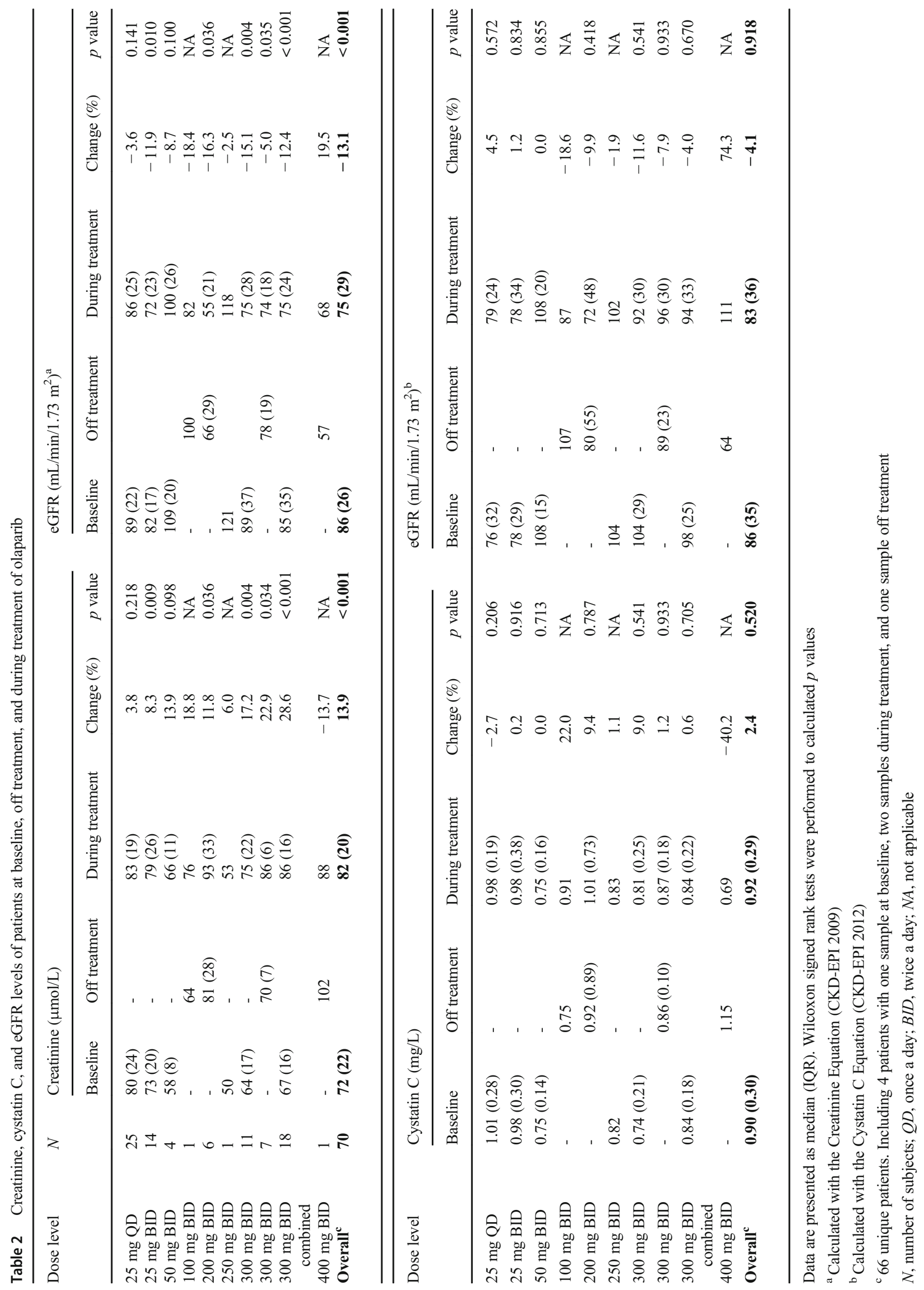



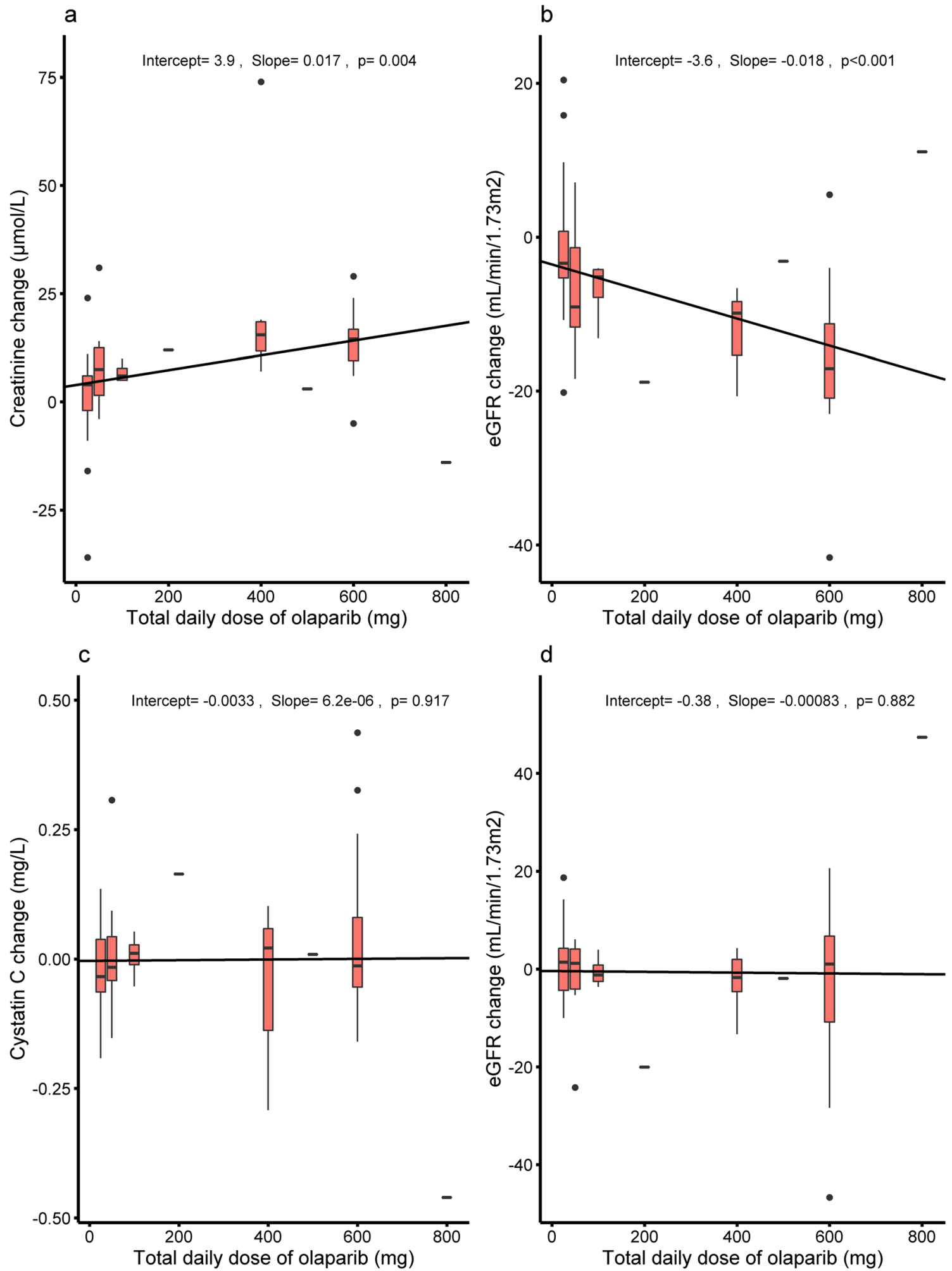

Fig. 2 The absolute change in creatinine (a), creatinine-derived eGFR (b), cystatin C (c), and cystatin C-derived eGFR (d) stratified by total daily dose of olaparib. Boxplots are given for each dose level where the middle line represents the median with margins representing the 25 th and

$40 \%$, and $+74 \%$, respectively) was hospitalized at the moment the "off treatment" sample was collected. The

75th percentiles. A dash is shown in case a single patient represents the dose level. Outliers are represented as dots. Linear regression was performed to calculate the intercept, slope and $p$ value

patient was suspicious for a bacterial infection and had fever, nausea, was vomiting, and had a CRP of $27 \mathrm{mg} / \mathrm{L}$. 

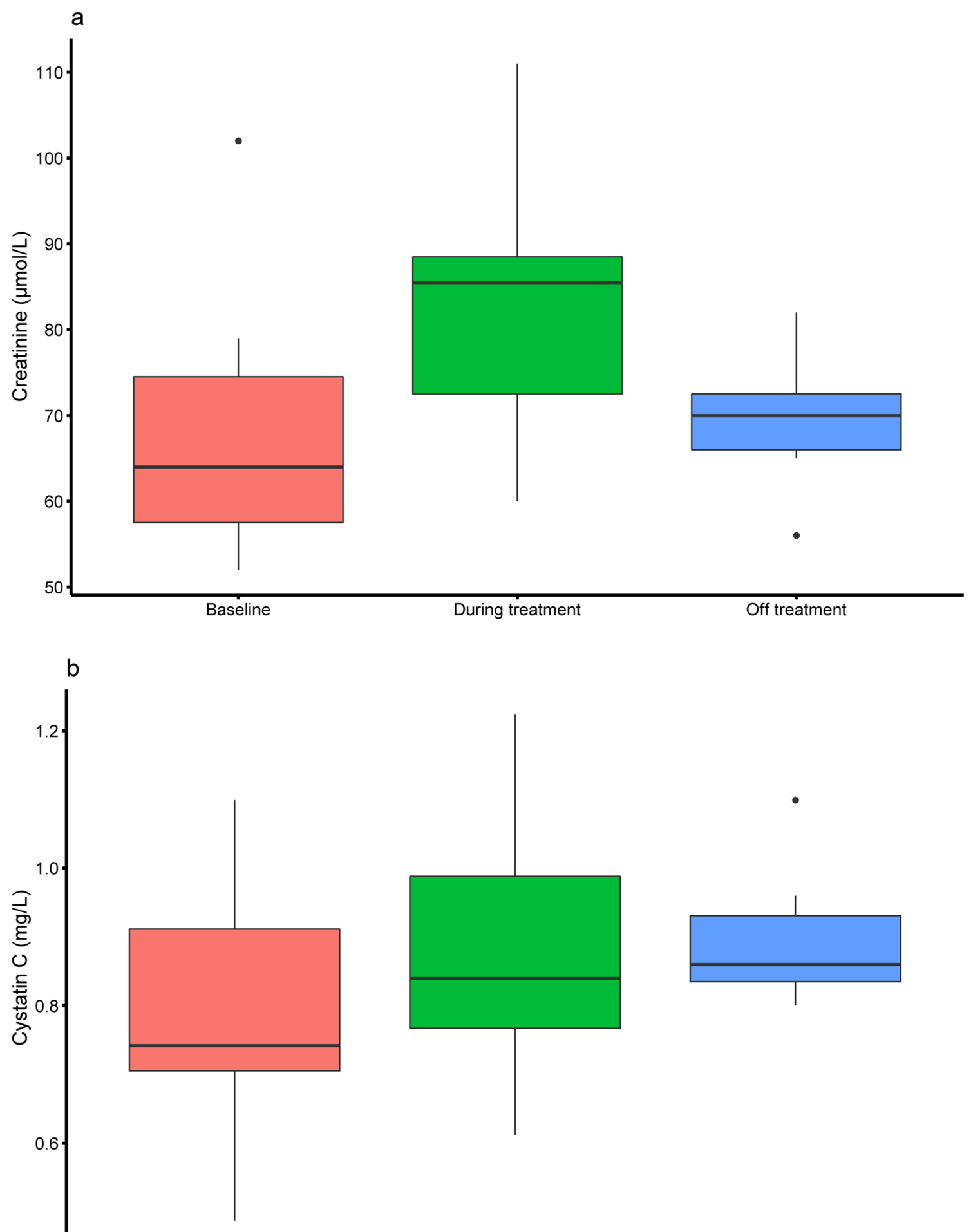

Baseline

During treatment

Off treatment

Fig. 3 Creatinine (a) and cystatin C (b) levels of patients treated with olaparib $300 \mathrm{mg}$ BID at baseline $(n=11)$, during treatment $(n=18)$, and after (temporary) treatment discontinuation $(n=7)$. Boxplots are given

\section{Discussion}

Increases in creatinine levels have been associated with olaparib administration [2]. An increase in creatinine levels where the middle line represents the median with margins representing the 25 th and 75 th percentiles

can arise from damage to the nephrons resulting in a reduced glomerular filtration rate and reduced creatinine clearance. Another explanation is a decrease in tubular secretion of creatinine caused by inhibition of the OCT2 and/or MATE1/2-K 
transporters by drugs. In vitro evaluation of olaparib predicted that olaparib inhibits the OCT2 and MATE1/2-K transporters at clinically relevant concentrations. Therefore, we hypothesized that the increases in creatinine are a result of inhibition of tubular secretion and not a reduction in GFR.

Significant increases in creatinine levels $(+14 \%)$ were observed during treatment of olaparib, which is lower than the + $23 \%$ reported in literature. However, we included patients at dose levels ranging from $25 \mathrm{mg}$ QD to $400 \mathrm{mg}$ BID. We observed a dose-dependent increase in creatinine levels, with an increase of $29 \%$ in patients treated at the recommended dose of olaparib monotherapy (300 mg BID). Paired samples were available between baseline and during treatment and during treatment and off treatment, but not between baseline and off treatment (except for 4 patients). However, creatinine levels at baseline, as well as off treatment, differ statistically significant from creatinine levels during treatment, which suggests the increases in creatinine to be reversible. Since inhibition is reversible, off treatment samples were combined with baseline samples to investigate the overall effect combining all dose levels.

Cystatin $\mathrm{C}$ levels were measured to investigate the mechanism behind these increases. Damage to nephrons resulting in a decreased GFR will be reflected in increased cystatin C levels, as well as in increased creatinine levels, both resulting in a decreased eGFR. However, no statistically significant changes in median cystatin $\mathrm{C}$ levels and median cystatin $\mathrm{C}-$ derived eGFRs were observed. Since cystatin $C$ is not influenced by tubular secretion, it is likely that olaparib does not cause glomerular injury, GFR is not changed following olaparib treatment, and the increase in creatinine is the result of inhibition of renal transporters by olaparib, which is reversible after treatment discontinuation.

Increases in creatinine levels were dose dependent and even observed at dose levels far below the recommended monotherapy dose. Apparently, low doses of olaparib are sufficient to cause inhibition of the renal transporters resulting in an increase in creatinine. The lowest dose level of $25 \mathrm{mg}$ QD had no significant effect on creatinine, while bidaily dosing did have an effect. Creatinine returns back to baseline after discontinuation of a reversible inhibitor of renal transporters $[7,15]$. So either the unbound olaparib concentration in blood is too low to cause sufficient inhibition of renal transporters or the once daily dose regimen allows creatinine to return to baseline levels within the dosing interval.

Not only GFR and tubular secretion can impact creatinine levels in blood. Creatinine levels can be affected by diet, malnutrition, and muscle mass, but also by drugs causing kidney damage [3]. Since oncology patients are complex patients using many drugs, comedication was taken into account. The highly nephrotoxic drug cisplatin was used by ten patients during olaparib treatment. Although the administered dose of cisplatin was low, the nephrotoxic effect of cisplatin increases with dose, frequency of administration, and cumulative dose [16] and therefore, cisplatin should be included in the analysis. Linear regression analysis showed no association between cisplatin on change in creatinine. If cisplatin had a significant effect on creatinine due to kidney damage, this would have been reflected in cystatin $\mathrm{C}$ levels, which was not the case. Cisplatin is excreted in urine which is mediated by the OCT and MATE transporters. Inhibition of OCT2 prevents accumulation and toxicity of cisplatin, but inhibition of MATE potentiates cisplatin-induced nephrotoxicity. Since olaparib inhibits both transporters, it is not clear if olaparib has protective or inductive effects [4].

While creatinine levels were increased during olaparib treatment in the majority of patients with no change in cystatin $\mathrm{C}$ levels, we did observe substantial changes in cystatin $\mathrm{C}$ levels along with creatinine levels in two patients. In one of these patients, a substantial difference between cystatin and creatinine-derived eGFR was noted, most likely explained by the inflammation status of this patient. The patient had an elevated CRP concentration of $27 \mathrm{mg} / \mathrm{L}$ during olaparib treatment. Inflammation measured by CRP is one of the factors independently positively associated with cystatin $\mathrm{C}$ levels and not with creatinine levels. Cystatin $\mathrm{C}$ could be a biomarker for inflammation and is also influenced by factors other than renal function [17].

Not only creatinine levels can be increased by olaparib treatment; another important consequence is the potential of drug-drug interactions (DDIs). Inhibition of the OCT2 transporter by olaparib can increase the exposure of drugs transported by the OCT2 transporter, which is, for example, observed for cimetidine co-administrated with metformin [18]. However, it should be investigated further if olaparib causes a clinically relevant increase in exposure of drugs transported by the OCT2 and/or MATE1/2-K transporters.

Our study has some limitations. First, the study had a retrospective design. We were dependent on samples stored in our biobank or pharmacy and baseline samples were not available from all patients. Time of administration of olaparib and time of blood withdrawal were variable in patients not enrolled in clinical trials. Creatinine concentrations can vary and will be maximal after the $t_{\max }$ of olaparib gradually returning to baseline until the next dose of olaparib is administrated [7]. Second, cystatin $C$ is not a perfect marker for renal function. Cystatin $\mathrm{C}$ can be affected by hyperthyroidism, steroid use, and inflammation. Therefore, exogenous markers such as iothalamate, iohexol, or inulin are the most accurate to measure GFR, but these compounds are invasive and expensive. Despite these limitations, the results are significant and convincing. Our study shows that olaparib treatment causes a dose-dependent, reversible increase in creatinine levels and has no effect on cystatin $\mathrm{C}$ levels. Therefore, we hypothesize that olaparib does not cause renal injury, has no effect on glomerular filtration, but affects tubular secretion of 
creatinine resulting in a decreased eGFR. Our observations are in line with a recently published study on the discrepancy between the calculated GFR using creatinine and the measured GFR using GFR scans in patients using PARP inhibitors, which empowers our hypothesis. In this study, a significant decline in creatinine-derived eGFR was found, while the eGFR from renal scans was nearly identical [19]. Here, we confirm these results with an additional and easily accessible biomarker for renal clearance and show the dose dependency of this effect.

Physicians should be aware that in patients taking olaparib, the eGFR based on creatinine can give an underestimation of renal function. This is especially important if the calculated eGFR drops below the level in which dose adjustments of drugs are made. For many drugs, this is below a creatinine clearance of 30 or $50 \mathrm{~mL} / \mathrm{min}$, but in some cases even below $80 \mathrm{~mL} / \mathrm{min}$. Based on our hypothesis, no dose adjustments are necessary for drugs eliminated by glomerular filtration, including olaparib itself. Dose adjustments made based on the decreased eGFR can result in underdosing of patients. An alternative marker such as cystatin $C$ should be used to accurately assess renal function in patients with moderate to severe renal function.

\section{Conclusion}

This study demonstrates that olaparib treatment leads to a significant, dose-dependent, and reversible increase in creatinine levels. Olaparib likely causes inhibition of renal transporters and does not affect GFR, since the cystatin C-derived eGFR was comparable before/off treatment and during treatment of olaparib. Physicians prescribing olaparib should be aware of the underestimation of renal function using the creatinine-derived eGFR and the potential for drug-drug interactions. It is advised to use an alternative renal marker such as cystatin $\mathrm{C}$ to accurately calculate eGFR.

Authors' contributions Conceptualization and methodology: M.A.C. Bruin, D. van den Broek, A.D.R. Huitema; formal analysis: M.A.C. Bruin; investigation: M.A.C. Bruin, C.M. Korse, B. van Wijnen; resources: C.M. Korse, B. van Wijnen, V.M.T. de Jong, S.C. Linn, B. van Triest, H. Rosing; writing - original draft: M.A.C. Bruin; writing review and editing: all authors; supervision: H. Rosing, J.H. Beijnen, D. van den Broek, A.D.R. Huitema.

Data availability The datasets generated during and/or analyzed during the current study are available from the corresponding author on reasonable request.

\section{Compliance with ethical standards}

Conflict of interest The authors declare that they have no conflicts of interest.
Ethics approval Institutional review board (IRB) approval was obtained for this study from the IRB of the NKI-AVL.

Consent to participate An additional informed consent was not required for this retrospective study in agreement with FEDERA (Federation of Dutch University Medical Centers), code of conduct for responsible use in human tissue and medical research (www.nfu.nl).

Consent for publication Not applicable.

Code availability Not applicable.

Open Access This article is licensed under a Creative Commons Attribution 4.0 International License, which permits use, sharing, adaptation, distribution and reproduction in any medium or format, as long as you give appropriate credit to the original author(s) and the source, provide a link to the Creative Commons licence, and indicate if changes were made. The images or other third party material in this article are included in the article's Creative Commons licence, unless indicated otherwise in a credit line to the material. If material is not included in the article's Creative Commons licence and your intended use is not permitted by statutory regulation or exceeds the permitted use, you will need to obtain permission directly from the copyright holder. To view a copy of this licence, visit http://creativecommons.org/licenses/by/4.0/.

\section{References}

1. US Food and Drug Administration (FDA) (2018) LYNPARZA (Olaparib): US prescribing information. https://www.accessdata. fda.gov/drugsatfda_docs/label/2018/208558s001lbl.pdf. Accessed 10 Oct 2019

2. Committee for Medicinal Products for Human Use (CHMP) European Medicines Agency (EMA) (2019) ANNEX I SUMMARY OF PRODUCT CHARACTERISTICS - Olaparib. https://www.ema.europa.eu/en/documents/product-information/ lynparza-epar-product-information_en.pdf. Accessed 10 Oct 2019

3. Levey A (1988) Serum creatinine and renal function. Annu Rev Med 39:465-490. https://doi.org/10.1146/annurev.med.39.1.465

4. Yonezawa A, Inui K (2011) Importance of the multidrug and toxin extrusion MATE/SLC47A family to pharmacokinetics, pharmacodynamics/toxicodynamics and pharmacogenomics. Br J Pharmacol 164:1817-1825. https://doi.org/10.1111/j.1476-5381. 2011.01394.x

5. Hilbrands LB, Artz MA, Wetzels JFM, Koene RAP (1991) Cimetidine improves the reliability of creatinine as a marker of glomerular filtration. Kidney Int 40:1171-1176. https://doi.org/ 10.1038/ki.1991.331

6. Naderer O, Nafziger AN, Bertino JS (1997) Effects of moderatedose versus high-dose trimethoprim on serum creatinine and creatinine clearance and adverse reactions. Antimicrob Agents Chemother 41:2466-2470. https://doi.org/10.1128/AAC.41.11. 2466

7. Chappell JC, Turner PK, Pak YA, Bacon J, Chiang AY, Royalty J, Hall SD, Kulanthaivel P, Bonventre JV (2019) Abemaciclib inhibits renal tubular secretion without changing glomerular filtration rate. Clin Pharmacol Ther 105:1187-1195. https://doi.org/10.1002/ cpt.1296

8. Dharnidharka VR, Kwon C, Stevens G (2002) Serum cystatin C is superior to serum creatinine as a marker of kidney function: a metaanalysis. Am J Kidney Dis 40:221-226. https://doi.org/10.1053/ ajkd.2002.34487 
9. Tenstad O, Roald AB, Grubb A, Aukland K (1996) Renal handling of radiolabelled human cystatin $\mathrm{C}$ in the rat. Scand J Clin Lab Invest 56:409-414. https://doi.org/10.3109/00365519609088795

10. McCormick A, Swaisland H (2017) In vitro assessment of the roles of drug transporters in the disposition and drug-drug interaction potential of olaparib. Xenobiotica 47:903-915. https://doi.org/10. 1080/00498254.2016.1241449

11. Rolfo C, de Vos-Geelen J, Isambert N, Molife LR, Schellens JHM, de Grève J, Dirix L, Grundtvig-Sørensen P, Jerusalem G, Leunen K, Mau-Sørensen M, Plummer R, Learoyd M, Bannister W, Fielding A, Ravaud A (2019) Pharmacokinetics and safety of olaparib in patients with advanced solid tumours and renal impairment. Clin Pharmacokinet 58:1165-1174. https://doi.org/10.1007/ s40262-019-00754-4

12. Inker LA, Schmid CH, Tighiouart H, Eckfeldt JH, Feldman HI, Greene T, Kusek JW, Manzi J, van Lente F, Zhang YL, Coresh J, Levey AS, CKD-EPI Investigators (2012) Estimating glomerular filtration rate from serum creatinine and cystatin C. N Engl J Med 367:20-29. https://doi.org/10.1056/NEJMoa1114248

13. R Core Team (2018) A language and environment for statistical computing. R Found Stat Comput Vienna, Austria https://www. R-project.org

14. Pattaro C, Riegler P, Stifter G, Modenese M, Minelli C, Pramstaller PP (2013) Estimating the glomerular filtration rate in the general population using different equations: effects on classification and association. Nephron Clin Pract 123:102-111. https://doi.org/10. $1159 / 000351043$
15. Chu X, Bleasby K, Chan GH, Nunes I, Evers R (2016) The complexities of interpreting reversible elevated serum creatinine levels in drug development: does a correlation with inhibition of renal transporters exist? Drug Metab Dispos 44:1498-1509. https://doi. org/10.1124/dmd.115.067694

16. Miller RP, Tadagavadi RK, Ramesh G, Reeves WB (2010) Mechanisms of cisplatin nephrotoxicity. Toxins (Basel) 2:2490 2518. https://doi.org/10.3390/toxins2112490

17. Knight EL, Verhave JC, Spiegelman D, Hillege HL, de Zeeuw D, Curhan GC, de Jong PE (2004) Factors influencing serum cystatin $\mathrm{C}$ levels other than renal function and the impact on renal function measurement. Kidney Int 65:1416-1421. https://doi.org/10.1111/j. 1523-1755.2004.00517.x

18. Somogyi A, Stockley C, Keal J, Rolan P, Bochner F (1987) Reduction of metformin renal tubular secretion by cimetidine in man. Br J Clin Pharmacol 23:545-551. https://doi.org/10.1111/j. 1365-2125.1987.tb03090.x

19. Zibetti Dal Molin G, Westin SN, Msaouel P, Gomes LM, Dickens A, Coleman RL (2020) Discrepancy in calculated and measured glomerular filtration rates in patients treated with PARP inhibitors. Int J Gynecol Cancer 30:89-93. https://doi.org/10.1136/ijgc-2019000714

Publisher's note Springer Nature remains neutral with regard to jurisdictional claims in published maps and institutional affiliations. 